\title{
DOE/ER/40272--296
}

\section{Toward the $M(F)$-Theory Embedding of Realistic Free-Fermion Models}

P. Berglund ${ }^{1}$, J. Ellis ${ }^{2}$, A.E. Faraggi ${ }^{3}$, D.V. Nanopoulos ${ }^{4}$, and Z. Qiu ${ }^{3}$

1 Institute for Theoretical Physics, University of California, Santa Barbara, CA 93106, USA

2 Theory Division, CERN, CH-1211 Geneva, Switzerland

${ }^{3}$ Institute for Fundamental Theory, Department of Physics, University of Florida, Gainesville, FL 32611, USA

${ }^{4}$ Center for Theoretical Physics, Dept. of Physics, Texas A \& $M$ University, College Station, TX 77843-4242, USA,

Astroparticle Physics Group, Houston Advanced Rescarch Center (HARC), The Mitchell Campus, Woodlands, TX 77381. USA, and

Academy of Athens, Chair of Theoretical Physics, Division of Natural Sciences, 28 Panepistimiou Avenue, Athens 106\%9, Greece.

\begin{abstract}
We construct a Landau-Ginzburg model with the same data and symmetries as a $Z_{2} \times Z_{2}$ orbifold that corresponds to a class of realistic free-fermion models. Within the class of interest, we show that this orbifolding connects between different $Z_{2} \times Z_{2}$ orbifold models and commutes with the mirror symmetry. Our work suggests that duality symmetries previously discussed in the context of specific $M$ and $F$ theory compactifications may be extended to the special $Z_{2} \times Z_{2}$ orbifold that characterizes realistic free-fermion models.
\end{abstract}

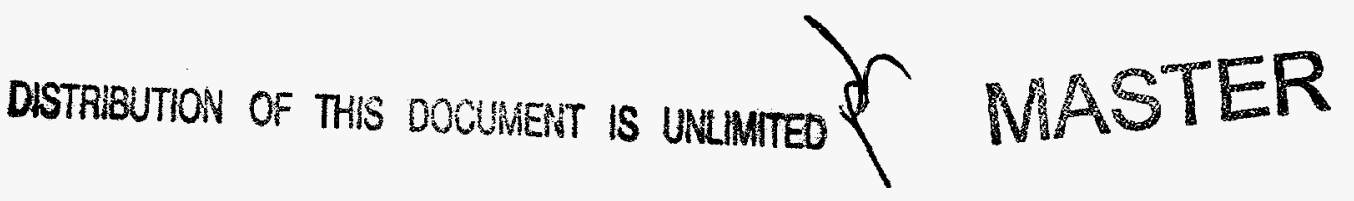




\section{Introduction}

The rapid recent development of tools based on duality for the analysis of superstring models has opened up a new range of possibilities beyond the weak-coupling models studied earlier. Moreover, it has been suggested that matching the bottom-up estimation of the unification scale based on low-energy data with the top-down estimate based on string theory is easiest in a strong-coupling limit of heterotic string [1]. These developments prompt a reformulation of the string model-building approaches pioneered previously.

Among phenomenological superstring models, those $[2,3,4,5,6]$ derived in the free-fermion formulation [7] have been taken the furthest in attempts to construct a semi-realistic string model that can incorporate the Standard Model of particle physics [8]. This may reflect the fact that the true string vacuum, even if stronglycoupled and still elusive, may share some properties with the models constructed thus far. In order to appreciate the realistic nature of the free-fermion models, it is useful to recall their underlying structure. These free-fermion models may be phrased in terms of an underlying $Z_{2} \times Z_{2}$ orbifold compactification, by going to the point in the Narain moduli space [9] where all the internal compactified dimensions can be represented as free world-sheet fermions. Three-generation models then arise naturally, due to the structure of the $Z_{2} \times Z_{2}$ orbifold. Furthermore, an important property of the free-fermion models is the standard $S O(10)$ embedding of the weak hypercharge, which ensures natural consistency between the experimental values for $\alpha_{s}\left(M_{Z}\right)$ and $\sin ^{2} \theta_{W}\left(M_{Z}\right)$. Moreover, these models may also avoid the non-universal contributions to soft supersymmetry-breaking scalar masses in the MSSM that may arise from the moduli sector or from charges under an anomalous $U(1)$ symmetry, leading to well known problems with flavor-changing neutral currents.- Due to their $Z_{2} \times Z_{2}$ orbifold structure, in the free-fermion models these contributions may be universal in flavor [10], enhancing the phenomenological viability of these models. Finally, the symmetries related to the $Z_{2} \times Z_{2}$ orbifold structure, which are not present in ordinary GUTs, may be important for understanding the proton stability $[11,12]$.

The above remarks motivate the need to understand better the general structure of the realistic free-fermion models, particularly in the context of the recent progress in understanding the nonperturbative generalization of weakly-coupled string theories [13]. A key problem is to study how the advances in understanding nonperturbative aspects of string theories may be applied to the free-fermion models. The purpose of this paper is to take a first step towards this goal.

Most of the discussion on nonperturbative generalizations of string compactifications are in the context of geometrical compactifications, i.e., Calabi-Yau manifolds including $K 3$ and toroidal compactifications. The free-fermion models are however constructed at a fixed point in the moduli space, and the direct notion of a compactified manifold is lost. Therefore, a desirable step in advancing our understanding of 


\section{DISCLAIMER}

This report was prepared as an account of work sponsored by an agency of the United States Government. Neither the United States Government nor any agency thereof, nor any of their employees, makes any warranty, express or implied, or assumes any legal liability or responsibility for the accuracy, completeness, or usefulness of any information, apparatus, product, or process disclosed, or represents that its use would not infringe privately owned rights. Reference herein to any specific commercial product, process, or service by trade name, trademark, manufacturer, or otherwise does not necessarily constitute or imply its endorsement, recommendation, or favoring by the United States Government or any agency thereof. The views and opinions of authors expressed herein do not necessarily state or reflect those of the United States Government or any agency thereof. 


\section{DISCLAIMER}

Portions of this document may be illegible electronic image products. Images are produced from the best available original document. 
free-fermion models to the nonperturbative regime is to find the Calabi-Yau three fold which correspond to the compactification underlying the free-fermion models. A well known connection exist between Calabi-Yau compactifications and their realization in terms of Landau-Ginzburg potentials. In this paper we therefore seek to find a Landau-Ginzburg model that may correspond to the $Z_{2} \times Z_{2}$ orbifold model at the free-fermion point in the Narain moduli space. We will also find a natural candidate in terms of a Calabi-Yau orbifold. In the toroidal language, the $Z_{2} \times Z_{2}$ orbifold model at the free-fermion point differs from the one traditionally discussed in the literature. The difference is that at the free-fermion point the symmetry of the compactified lattice is enhanced to $S O(12)$, whereas for the traditional $Z_{2} \times Z_{2}$ orbifold one compactifies on a $\left(T_{2}\right)^{3}$ lattice with $S O(4)^{3}$ symmetry. This distinction results in the two models having different Euler characteristics. Whereas the $Z_{2} \times Z_{2}$ orbifold on the $S O(4)^{3}$ lattice produces $\left(h_{11}, h_{21}\right)=(51,3)$, that on the $S O(12)$ lattice produces $\left(h_{11}, h_{21}\right)=(27,3)[14,12]$. Therefore, our aim here is to find a Landau-Ginzburg realization of the $Z_{2} \times Z_{2}$ orbifold on $S O(12)$ lattice with $\left(h_{11}, h_{21}\right)=(27,3)$. In the course of seeking this model we also examine some of the features of the Landau-Ginzburg formalism. As is well known, the LandauGinzburg models $[15,16]$ are obtained by specifying a superpotential $W$ and modding by some discrete symmetries of the potential. To construct a consistent string theory from the Landau-Ginzburg potential one has to project on states with integral leftand right-moving charges and to include in the spectrum the states from the twisted sector. One twist that must be included for obtaining a consistent string theory is that by the scaling symmetry of the Landau-Ginzburg potential. The novel feature that we will examine here is orbifolding of Landau-Ginzburg potentials by a twist that closes on the scaling symmetry. This is therefore an extended method for obtaining consistent Landau-Ginzburg models. In the course of searching for our specific Landau-Ginzburg orbifold, we will also find new connections between the different Landau-Ginzburg models reminiscent of the mirror-symmetry phenomenon.

\section{The Free-Fermion $Z_{2} \times Z_{2}$ Orbifold}

In the free-fermion formulation [7], a model is defined by a set of boundary condition basis vectors, together with the related one-loop GSO projection coefficients, that are constrained by the string consistency constraints. The basis vectors, $b_{k}$, span a finite additive group, $\Xi=\sum_{k} n_{k} b_{k}$ where $n_{k}=0, \cdots, N_{z_{k}}-1$. The physical states in the Hilbert space of a given sector $\alpha \in \Xi$ are obtained by acting on the vacuum with bosonic and fermionic operators and by applying the generalized GSO projections. The $U(1)$ charges $Q(f)$ corresponding to the unbroken Cartan generators of the four-dimensional gauge group, which are in one-to-one correspondence with the $U(1)$ currents $f^{*} f$ for each complex fermion $f$, are given by:

$$
Q(f)=\frac{1}{2} \alpha(f)+F(f)
$$


where $\alpha(f)$ is the boundary condition of the world-sheet fermion $f$ in the sector $\alpha$, and $F_{\alpha}(f)$ is a fermion-number operator that takes the value +1 for each mode of $f$, and the value -1 for each mode of $f^{*}$, if $f$ is complex. For periodic fermions, which have $\alpha(f)=1$, the vacuum must be a spinor in order to represent the Clifford algebra of the corresponding zero modes. For each periodic complex fermion $f$, there are two degenerate vacua $|+\rangle,|-\rangle$, annihilated by the zero modes $f_{0}$ and $f_{0}{ }^{*}$, respectively, and with fermion numbers $F(f)= \pm 1$.

Realistic models in this free-fermion formulation are generated by a suitable choice of boundary-condition basis vectors for all world-sheet fermions, which may be constructed in two stages. The first stage consists of the NAHE set $[2,17]$ of five boundary condition basis vectors, $\left\{\mathbf{1}, S, b_{1}, b_{2}, b_{3}\right\}$. After generalized GSO projections over the NAHE set, the residual gauge group is $S O(10) \times S O(6)^{3} \times E_{8}$ with $N=1$ space-time supersymmetry ${ }^{*}$. The space-time vector bosons that generate the gauge group arise from the Neveu-Schwarz sector and from the sector $1+b_{1}+b_{2}+b_{3}$. The Neveu-Schwarz sector produces the generators of $S O(10) \times S O(6)^{3} \times S O(16)$. The sector $1+b_{1}+b_{2}+b_{3}$ produces the spinorial 128 of $S O(16)$ and completes the hidden-sector gauge group to $E_{8}$. The vectors $b_{1}, b_{2}$ and $b_{3}$ correspond to the three twisted sectors in the corresponding orbifold formulation, and produce 48 spinorial 16-dimensional representations of $S O(10)$, sixteen each from the sectors $b_{1}, b_{2}$ and $b_{3}$.

The second stage of the basis construction consists of adding three more basis vectors to the NAHE set, corresponding to Wilson lines in the orbifold formulation, whose general forms are constrained by string consistency conditions such as modular invariance, as well as by space-time supersymmetry. These three additional vectors are needed to reduce the number of generations to three, one from each of the sectors $b_{1}, b_{2}$ and $b_{3}$. The details of the additional basis vectors distinguish between different models and determine their phenomenological properties. This construction produces a large number of three-generation models with different phenomenological characteristics, some of which are especially appealing. This class of models corresponds to $Z_{2} \times Z_{2}$ orbifold compactification at the maximally-symmetric point in the Narain moduli space [9]. The emergence of three generations is correlated with the underlying $Z_{2} \times Z_{2}$ orbifold structure. Detailed studies of specific models have revealed that these models may explain the qualitative structure of the fermion mass spectrum [18] and could form the basis of a realistic superstring model. We refer the interested reader to several review articles which summarize the phenomenological studies of this class of models [8].

The NAHE set is common in a large class of three-generation free-fermion models, and its characteristic properties are reflected in many of the phenomenological properties of these models. Therefore, it is important to extend our understanding of this basic set and its properties. This set corresponds to a $Z_{2} \times Z_{2}$ orbifold compacti-

*The vector $S$ in this NAHE set is the supersymmetry generator, and the superpartners of the states from a given sector $\alpha$ are obtained from the sector $S+\alpha$. 
fication of the weakly-coupled ten-dimensional heterotic string, and the basis vectors $b_{1}, b_{2}$ and $b_{3}$ correspond to the three twisted sectors of these orbifold models. To see this correspondence, we add to the NAHE set the basis vector

$$
X=(0, \cdots, 0 \mid \underbrace{1, \cdots, 1}_{\bar{\psi}^{1}, \cdots, 5, \bar{\eta}^{1,2,3}}, 0, \cdots, 0) .
$$

with the following choice of generalized GSO projection coefficients:

$$
c\left(\begin{array}{l}
X \\
\mathbf{b}_{j}
\end{array}\right)=-c\left(\begin{array}{l}
X \\
S
\end{array}\right)=c\left(\begin{array}{c}
X \\
\mathbf{1}
\end{array}\right)=+1 .
$$

This set of basis vectors produces models with an $S O(4)^{3} \times E_{6} \times U(1)^{2} \times E_{8}$ gauge group and $N=1$ space-time supersymmetry. The matter fields include 24 generations in 27 representations of $E_{6}$, eight from each of the sectors $b_{1} \oplus b_{1}+X$, $b_{2} \oplus b_{2}+X$ and $b_{3} \oplus b_{3}+X$. Three additional 27 and $\overline{27}$ pairs are obtained from the Neveu-Schwarz $\oplus X$ sector.

The subset of basis vectors

$$
\left\{1, S, X, I=\mathbf{1}+b_{1}+b_{2}+b_{3}\right\}
$$

generates a toroidally-compactified model with $N=4$ space-time supersymmetry and $S O(12) \times E_{8} \times E_{8}$ gauge group. The same model is obtained in the geometric (bosonic) language by constructing the background fields which produce the $S O(12)$ Narain lattice $[9,14]$, taking the metric of the six-dimensional compactified manifold to be the Cartan matrix of $S O(12)$ :

$$
g_{i j}=\left(\begin{array}{cccccc}
2 & -1 & 0 & 0 & 0 & 0 \\
-1 & 2 & -1 & 0 & 0 & 0 \\
0 & -1 & 2 & -1 & 0 & 0 \\
0 & 0 & -1 & 2 & -1 & -1 \\
0 & 0 & 0 & -1 & 2 & 0 \\
0 & 0 & 0 & -1 & 0 & 2
\end{array}\right)
$$

and the antisymmetric tensor

$$
b_{i j}= \begin{cases}g_{i j} & ; i>j \\ 0 & ; i=j \\ -g_{i j} & ; i<j\end{cases}
$$

When all the radii of the six-dimensional compactified manifold are fixed at $R_{I}=\sqrt{2}$, it is easily seen that the left- and right-moving momenta

$$
P_{R, L}^{I}=\left[m_{i}-\frac{1}{2}\left(B_{i j} \pm G_{i j}\right) n_{j}\right] e_{i}^{I^{*}}
$$


reproduce all the massless root vectors in the lattice of $S O(12)$, where in (2.7) the $e^{i}=\left\{e_{i}^{I}\right\}$ are six linearly-independent vectors normalized: $\left(e_{i}\right)^{2}=2$. The $e_{i}^{I^{*}}$ are dual to the $e_{i}$, and $e_{i}^{*} \cdot e_{j}=\delta_{i j}$. The momenta $P^{I}$ of the compactified scalars in the bosonic formulation can be seen to coincide with the $U(1)$ charges of the unbroken Cartan generators of the four dimensional gauge group (2.1).

Adding the two basis vectors $b_{1}$ and $b_{2}$ to the set (2.4) corresponds to the $Z_{2} \times Z_{2}$ orbifold model with standard embedding. The fermionic boundary conditions are translated in the bosonic language to twists on the internal dimensions and shifts on the gauge degrees of freedom. Starting from the Narain model with $S O(12) \times E_{8} \times E_{8}$ symmetry [9], and applying the $Z_{2} \times Z_{2}$ twisting on the internal coordinates, we then obtain the orbifold model with $S O(4)^{3} \times E_{6} \times U(1)^{2} \times E_{8}$ gauge symmetry. There are sixteen fixed points in each twisted sector, yielding the 24 generations from the three twisted sectors mentioned above. The three additional pairs of 27 and $\overline{27}$ are obtained from the untwisted sector. This orbifold model exactly corresponds to the freefermion model with the six-dimensional basis set $\left\{\mathbf{1}, S, X, I=\mathbf{1}+b_{1}+b_{2}+b_{3}, b_{1}, b_{2}\right\}$. The Euler characteristic of this model is 48 with $h_{11}=27$ and $h_{21}=3$.

This $Z_{2} \times Z_{2}$ orbifold, corresponding to the extended NAHE set at the core of the realistic free-fermion models, differs from the one which has usually been examined in the literature [25]. In that orbifold model, the Narain lattice is $S O(4)^{3}$, yielding a $Z_{2} \times Z_{2}$ orbifold model with Euler characteristic equal to 96 , or 48 generations, and $h_{11}=51, h_{21}=3$.

In more realistic free-fermion models, the vector $X$ is replaced by the vector $2 \gamma$ with periodic boundary conditions for $\left\{\bar{\psi}^{1, \cdots, 5}, \bar{\eta}^{1}, \bar{\eta}^{2}, \bar{\eta}^{3}, \bar{\phi}^{1, \cdots, 4}\right\}$ and anti-periodic for all the other world-sheet fermions. This modification has the consequence of producing a toroidally-compactified model with $N=4$ space-time supersymmetry and gauge group $S O(12) \times S O(16) \times S O(16)$. The $Z_{2} \times Z_{2}$ twisting breaks the gauge symmetry to $S O(4)^{3} \times S O(10) \times U(1)^{3} \times S O(16)$. The orbifold twisting still yields a model with 24 generations, eight from each twisted sector, but now the generations are in the chiral 16 representation of $S O(10)$, rather than in the 27 of $E_{6}$. The same model can be realized with the set $\left\{\mathbf{1}, S, X, I=\mathbf{1}+b_{1}+b_{2}+b_{3}, b_{1}, b_{2}\right\}$, projecting out the $16 \oplus \overline{16}$ from the sector $X$ by taking

$$
c\left(\begin{array}{c}
X \\
I
\end{array}\right) \rightarrow-c\left(\begin{array}{c}
X \\
I
\end{array}\right) .
$$

This choice also projects out the massless vector bosons in the 128 of $S O(16)$ in the hidden-sector $E_{8}$ gauge group, thereby breaking the $E_{6} \times E_{8}$ symmetry to $S O(10) \times$ $U(1) \times S O(16)$. This analysis confirms that the $Z_{2} \times Z_{2}$ orbifold on the $S O(12)$ Narain lattice is indeed at the core of the realistic free-fermion models. 


\section{Landau-Ginzburg Construction}

Our aim in this section is to find the Landau-Ginzburg potentials that produce the same data as the free-fermion $Z_{2} \times Z_{2}$ orbifold. We will also find a realization in terms of a Calabi-Yau orbifold. Let us briefly summarize the main steps in the construction of Landau-Ginzburg string models [19]. The $N=2$ Landau-Ginzburg models are defined by a nondegenerate quasi-homogeneous superpotential $W$ of degree $d$,

$$
W\left(\lambda^{n_{i}} X_{i}\right)=\lambda^{d} W\left(X_{i}\right)
$$

where $X_{i}$ are chiral superfields, $q_{i}=n_{i} / d$ are their left and right charges under the $U(1)_{J_{0}}$ current of the $N=2$ algebra, and the central charge is given by $c=$ $3 \sum_{i}\left(1-2 q_{i}\right)$. For a string vacuum with space-time supersymmetry we project to integral $U(1)$ charges by taking the orbifold $W\left(X_{i}\right) / j$, where $j=e^{i 2 \pi J_{0}}$, and need to include the twisted sectors in order to respect modular invariance. We can take other twistings of the original Landau-Ginzburg potential by taking the orbifold $W / G$, where $G$ is a symmetry group of the non-renormalized $N=2$ superpotential $W\left(X_{i}\right)$, and can be an arbitrary subgroup of the full group of symmetries of $W$. The complete Hilbert space in the orbifold Landau-Ginzburg theory, $\mathcal{H}=\oplus P_{h} \mathcal{H}^{h}, h \in G$ then contains all the states from the untwisted and twisted sectors, subject to the projection $g|\chi\rangle=|\chi\rangle$ for every $g \in H$, where $H$ is the center of $G$. The states in the $(c, c)$ ring (the (NS,NS) untwisted sector) are built from the NS vacuum by monomials in the chiral superfields $X_{i}$ modulo setting $\partial_{i} W=0$, and are thus written as,

$$
\prod_{i}\left(X_{i}\right)^{\left(\ell_{i}\right)}|0\rangle
$$

subject to the condition $\sum_{i} \ell_{i} q_{i} \in Z$ and $\partial W / \partial X_{i}=0$. In the sum over the twisted sectors we have to include all the sectors up to inequivalent classes of $G$. We remark that in obtaining the $Z_{2} \times Z_{2}$ Landau-Ginzburg model with $\left(h_{11}, h_{21}\right)=(27,3)$ we will use a twist that closes on the scaling projection $j$. That is, for example, $g^{2}=j^{2}$. The sum over the twisted sectors then excludes the sectors containing $g^{2}$ and $g^{3}$, which are equivalent to $j^{2}$ and $j^{2} g$.

In the twisted sectors the chiral superfields $X_{i}$ are twisted by $X_{i}(2 \pi)=h_{i}^{j} X_{j}(0)$. The chiral superfields are rotated to a basis in which $h$ is diagonal $h=\delta_{i}^{J} \exp \left(2 \pi i \Theta_{i}^{h}\right)$. The charges and degeneracies in the twisted sectors are then obtained by computing the charge of the lowest-charge state in the $h$-twisted Ramond sector, $|0\rangle_{R}^{h}$, given by

$$
\left(\begin{array}{l}
J_{0} \\
\bar{J}_{0}
\end{array}\right)|0\rangle_{R}^{h}=\left( \pm \sum_{\Theta_{i}^{h} \notin Z}\left(\Theta_{i}^{h}-\left[\Theta_{i}^{h}\right]-1 / 2\right)+\sum_{\Theta_{i}^{h} \in Z}\left(q_{i}-1 / 2\right)\right)|0\rangle_{R}^{h}
$$

where $[x]$ denotes the greatest integer smaller than $x$, and acting on the Ramond ground states with the untwisted fields

$$
\prod_{\Theta_{i}^{h} \in Z}\left(X_{i}\right)^{\left(\ell_{i}\right)}|0\rangle_{R}^{h}
$$


to produce the twisted integrally charged states. Using spectral flow on the left and right charges, with $(c / 6 ; c / 6)$, we obtain the charges in the $(c, c)$ ring of the twisted sectors. At the final step the projections imposed by all $g \in G$ are applied to produce the modular-invariant spectrum.

We now turn to the class of Landau-Ginzburg models that reproduces the data of the desired orbifold models. It is well known that the $T^{2}$ torus has the polynomial representation

$$
W=X_{1}^{4}+X_{2}^{4}+X_{3}^{2}
$$

modded by the $Z_{4}$ scaling symmetry. Note that the complex structure of the torus is fixed, $\tau=i$ or else the torus would not have a $Z_{4}$ symmetry ${ }^{*}$. Here the field $X_{3}$ is a trivial superfield as its conformal charge is zero, and is used to mimic the geometrical representation of $T^{2}$ for $W=0$. The starting point for our construction is therefore the holomorphic superpotential given by

$$
W=X_{1}^{4}+X_{2}^{4}+X_{3}^{2}+X_{4}^{4}+X_{5}^{4}+X_{6}^{2}+X_{7}^{4}+X_{8}^{4}+X_{9}^{2}
$$

which is a just a triple sum of the superpotential in (3.5) and $X_{3,6,9}$ are again trivial superfields. The superpotential in (3.6) correspond to a super-conformal field theory with $c=9$.

In order to construct a consistent string vacuum we need to consider the LandauGinzburg-orbifold $W / j$ where $j$ is the scaling symmetry

$$
Z_{4}^{j}:\left(X_{1}, \ldots, X_{9}\right) \rightarrow\left(i X_{1}, i X_{2},-X_{3}, i X_{4}, i X_{5},-X_{6}, i X_{7}, i X_{8},-X_{9}\right) .
$$

Let us denote this Landau-Ginzburg orbifold $\mathcal{M}$. The next stage in the construction is to mod out the superpotential by $Z_{2}$ and $Z_{4}$ symmetries. The spectrum is then obtained by analyzing the untwisted and twisted sectors, as outlined above. We note that the superpotential (3.6) (modulo the trivial superfield $X_{3,6,9}$ ) has a large symmetry group $Z_{4}^{6}$, where each superfield can be rotated separately by a $Z_{4}$ twist.

Rather than considering a Landau-Ginzburg orbifold corresponding to the $Z_{2} \times Z_{2}$ orbifold model with $\left(h_{11}, h_{21}\right)=(51,3)$ we will first construct the mirror model with $\left(h_{11}, h_{21}\right)=(3,51)^{\dagger}$. To obtain the mirror we take $\mathcal{M} /\left(Z_{2}^{A} \times Z_{2}^{B}\right)$ where

$$
\begin{aligned}
& Z_{2}^{A}:\left(X_{1}, \cdots, X_{9}\right) \rightarrow\left(X_{1}, X_{2}, X_{3},-X_{4},-X_{5}, X_{6},-X_{7},-X_{8}, X_{9}\right) ; \\
& Z_{2}^{B}:\left(X_{1}, \cdots, X_{9}\right) \rightarrow\left(-X_{1},-X_{2}, X_{3},-X_{4},-X_{5}, X_{6}, X_{7}, X_{8}, X_{9}\right) .
\end{aligned}
$$

This orbifold contains $30(1,1)$ states from the untwisted sector, one from each of the twisted sectors $\Theta^{g}$, and six from each of the twisted sectors $\Theta^{j^{2} g}$, where $g=$ $\left(Z_{2}^{A}, Z_{2}^{B}, Z_{2}^{A} \otimes Z_{2}^{B}\right)$, making a total of $51(1,1)$ states. It contains three $(-1,1)$ states from each of the twisted sectors $\Theta^{j g}$, thus reproducing the data of the $Z_{2} \times Z_{2}$ orbifold

${ }^{*}$ The Landau-Ginzburg potential for a generic torus is given by $W=X_{1}^{6}+X_{2}^{3}+X_{3}^{2}$.

${ }^{\dagger}$ Since the Landau-Ginzburg orbifold is just a quotient of a product of minimal models, mirror symmetry can be shown at the level of conformal field theory [20]. 
on the $S O(4)^{3}$ lattice. We note that the cyclic permutation symmetry between the twisted and untwisted spectrum, the characteristic property of the $Z_{2} \times Z_{2}$ orbifold, is exhibited in this model.

The mirror of the $Z_{2} \times Z_{2}$ Landau-Ginzburg orbifold model, with $\left(h_{11}, h_{21}\right)=$ $(3,27)$, can be reproduced in several ways. One option is to take the twist

$$
\begin{aligned}
& Z_{4}^{A}:\left(X_{1}, \cdots, X_{9}\right) \rightarrow\left(X_{1}, X_{2}, X_{3}, i X_{4},-i X_{5}, X_{6}, i X_{7},-i X_{8}, X_{9}\right) \\
& Z_{4}^{B}:\left(X_{1}, \cdots, X_{9}\right) \rightarrow\left(i X_{1},-i X_{2}, X_{3}, i X_{4},-i X_{5}, X_{6}, X_{7}, X_{8}, X_{9}\right) .
\end{aligned}
$$

and the three trivial superfields are kept fixed, i.e. $\left(X_{3}, X_{6}, X_{9}\right) \rightarrow\left(X_{3}, X_{6}, X_{9}\right)$. This choice however mixes the action of the $Z_{4}$ and $Z_{2} \times Z_{2}$ twists. Therefore, the cyclic permutation symmetry of the $Z_{2} \times Z_{2}$ orbifold is not transparent with this choice. A more elegant choice is obtained by taking the $Z_{2}^{A} \times Z_{2}^{B}$ twist of (3.8) and adding the following $Z_{4}$ twist,

$$
Z_{4}^{w}:\left(X_{1}, \cdots, X_{9}\right) \rightarrow\left(i X_{1},-i X_{2}, X_{3}, i X_{4},-i X_{5}, X_{6}, i X_{7},-i X_{8}, X_{9}\right) \text {. }
$$

Here again the trivial superfields are kept fixed. This twist closes on the scaling symmetry, $w^{2}=j^{2}$. Therefore, in summing over the twisted spectrum we only have to sum up to inequivalent classes of the twist group. That is, twisted sectors that contain $w^{2}$ or $w^{3}$ are excluded as they are equivalent to $j^{2}$ and $j w^{2}$.

An alternative way of obtaining the same model is to note that the $Z_{4}$ twist in (3.10) acts only as a $Z_{2}$ modding, as it closes on the $Z_{4}$ scaling projection. Thus, the $Z_{2}$ quotient is generated by taking the product $Z_{4}^{j} \otimes Z_{4}^{w}$. Then, taking the $Z_{2}^{A} \times Z_{2}^{B}$ twist of eq. (3.8) and adding the above $Z_{2}$ twist:

$$
Z_{2}^{w}:\left(X_{1}, \ldots, X_{9}\right) \rightarrow\left(-X_{1}, X_{2},-X_{3},-X_{4}, X_{5},-X_{6},-X_{7}, X_{8},-X_{9}\right),
$$

we have the Landau-Ginzburg orbifold $\mathcal{M} /\left(Z_{2}^{A} \times Z_{2}^{B} \times Z_{2}^{\omega}\right)$. Here the three trivial superfields are twisted by the $Z_{2}^{w}$ twist. We then obtain in this Landau-Ginzburg orbifold model $18(1,1)$ states from the untwisted sector, one $(1,1)$ state from each of the sectors $\Theta^{g}$ with $g=\left(Z_{2}^{A}, Z_{2}^{B}, Z_{2}^{A} \otimes Z_{2}^{B}\right)$, and two $(1,1)$ states from each of the twisted sectors $\Theta^{j^{2} g}$, producing a total of $27(1,1)$ states, as desired. Three $(-1,1)$ states are obtained, one from each of the twisted sectors $\Theta^{j g}$, thus reproducing the data of the $Z_{2} \times Z_{2}$ orbifold on the $S O(12)$ lattice. We note that the cyclic permutation symmetry between the twisted sectors is retained in this model. In fact, the model is seen to be obtainable from the $(51,3) Z_{2} \times Z_{2}$ Landau-Ginzburg orbifold model by the action of the $Z_{4}^{w}$ twist (3.10), or by the action of the $Z_{2}^{w}$ twist (3.11). The action of this twist is to reduce the number of $(1,1)$ untwisted states from 30 to 18 and the number of twisted states from the sectors $\Theta^{j^{2} g}$ from 18 to 6 , thus reducing the total number of $(1,1)$ states from 51 to 27 , as needed. The projection (3.11) is therefore seen to connect between the mirrors of the $(51,3)$ and $(27,3) Z_{2} \times Z_{2}$ Landau-Ginzburg orbifold models, respectively. 
So far we have only constructed the mirror models of the relevant $Z_{2} \times Z_{2}$ orbifolds. The Landau-Ginzburg orbifolds for the $Z_{2} \times Z_{2}$ orbifolds can, however, also be constructed. In particular, we note that the operation of the twist in (3.10) or (3.11) also works for these models. The $(51,3)$ model is obtained by the following twists, $\mathcal{M} /\left(Z_{4}^{A} \times Z_{4}^{B}\right)$ :

$$
\begin{aligned}
& Z_{4}^{A}:\left(X_{1}, \ldots, X_{9}\right) \rightarrow\left(X_{1}, X_{2}, X_{3}, i X_{4}, i X_{5}, X_{6}, i X_{7}, i X_{8}, X_{9}\right) \\
& Z_{4}^{B}:\left(X_{1}, \ldots, X_{9}\right) \rightarrow\left(i X_{1}, i X_{2}, X_{3}, i X_{4}, i X_{5}, X_{6}, X_{7}, X_{8}, X_{9}\right)
\end{aligned}
$$

This Landau-Ginzburg model has $3(1,1)$ states from the untwisted sector and 51 $(-1,1)$ states from the twisted sectors. This model therefore contains the spectrum. of the $(51,3)$ model. Just as for its mirror, the states from the (un)twisted sectors can be shown to satisfy the cyclic permutation symmetry characteristic of the $Z_{2} \times Z_{2}$ orbifold. Adding to this model the twist in (3.10) or (3.11) then produces the model with $3(1,1)$ states from the untwisted sector and $27(-1,1)$ states from the twisted sectors. The projection (3.10) (or (3.11)) is therefore observed to commute with the mirror symmetry operation. In the free-fermion $Z_{2} \times Z_{2}$ orbifold models the mirror symmetry transformation operates by taking the GSO coefficients $c\left(b_{i}, b_{j}\right) \rightarrow-c\left(b_{i}, b_{j}\right)$ and can be seen in the orbifold models to operate by the choice of the discrete torsion. The projection (3.11) may therefore be regarded as a new geometrical operation that connects between models with different Euler characteristic. It is of great interest to examine how this can be interpreted in a more geometrical language. We now turn to this point.

The projection (3.11), that we used to obtain the $(27,3) Z_{2} \times Z_{2}$ LandauGinzburg orbifold model from the $(51,3)$ one, can be given a very natural geometric interpretation. Consider $\left(T^{2}\right)^{3}$ given in terms of an intersection of three quartic equations, $p_{i}(x)=0$,

$$
p_{i}=\left(x_{1+3 i}\right)^{4}+\left(x_{2+3 i}\right)^{4}+\left(x_{3+3 i}\right)^{2}=0, \quad i=0,1,2 .
$$

The $Z_{2} \times Z_{2}$ orbifold with $\left(h_{11}, h_{21}\right)=(51,3)$ is then represented by

$$
\begin{aligned}
& Z_{2}^{1}:\left(x_{1}, \ldots, x_{9}\right) \rightarrow\left(x_{1}, x_{2}, x_{3},-x_{4}, x_{5}, x_{6},-x_{7}, x_{8}, x_{9}\right) \\
& Z_{2}^{2}:\left(x_{1}, \ldots, x_{9}\right) \rightarrow\left(-x_{1}, x_{2}, x_{3},-x_{4}, x_{5}, x_{6}, x_{7}, x_{8}, x_{9}\right) .
\end{aligned}
$$

There are 16 fixed tori in each of the twisted sectors due to $\left(Z_{2}^{1}, Z_{2}^{2}, Z_{1} \otimes Z_{2}^{2}\right)$, each contributing $16(1,1)$-forms. We then obtain the $(27,3) Z_{2} \times Z_{2}$ orbifold by the $Z_{2}^{w}$ quotient (3.11). We note that $Z_{2}^{w}$ is freely acting, i.e., there is no fixed-point set. Moreover, because of this fact, the new Calabi-Yau manifold has a non-trivial homotopy class, $\Pi_{1}=Z_{2}$.

The projection (3.10) introduced the novel feature that it closes on the scaling symmetry $j$ of the Landau-Ginzburg superpotential (3.6). We now elaborate further on this type of projection by studying the consequences of similar projections in 
two and four compactified dimensions. In the two-dimensional case, the LandauGinzburg superpotential is that of (3.5). In this case the requested projection is simply

$$
\left(X_{1}, X_{2}\right) \rightarrow\left(i X_{1},-i X_{2}\right)
$$

The original Landau-Ginzburg theory $W /\{j\}$ correspond to a $T_{2}$ torus and therefore one $(1,1)$ state from the untwisted sector, $X_{1}^{2} X_{2}^{2}$. In this case we note that the projection $g$ in (3.15) leaves this state invariant, and does not give rise to any additional states. Therefore in this case the Landau-Ginzburg theories $W /\{j\}$ and $W /\{j, w\}$ are identical. This in fact may have been expected as in one complex dimension the $T_{2}$ torus is the only Ricci-flat manifold.

We now turn to the four-dimensional case. The special projection is

$$
\left(X_{1}, X_{2}, X_{3}, X_{4}\right) \rightarrow\left(i X_{1},-i X_{2}, i X_{3},-i X_{4}\right)
$$

In this case the Landau-Ginzburg theory $W /\{j\}$ admits $19(1,1)$ states from the untwisted sector and one $(1,1)$ state from the sector $j^{2}$. In the Landau-Ginzburg theory $W /\{j, w\}$ the effect of the $w$ projection is to project eight of the $(1,1)$ states from the untwisted sector. The three twisted sectors $\left\{j^{2}, w, j^{2} w\right\}$ each give one $(1,1)$ state, whereas the two twisted sectors $\left\{j w, j^{3} w\right\}$ each give $3(1,1)$ states. Therefore, in this case we note that the two theories $W /\{j\}$ and $W /\{j, w\}$ again reproduce the same spectrum.

Finally, we turn to six dimensions, where the Landau-Ginzburg potential is given by (3.6). In this case the theory $W /\{j\}$ gives rise to $90(1,1)$ states from the untwisted sector. The theory $W /\{j, w\}$ with $w$ given by $(3.11)$ produces $48(1,1)$ states from the untwisted sector and three $(1,1)$ states from each of the twisted sectors $\left\{j w, j^{3} w\right\}$, giving a total of $54(1,1)$ states. Therefore we see that while in the one and two complex-dimensional cases the effect of the special $w$ projection is trivial, in the case of three complex dimensions new features are encountered which modify the original Landau-Ginzburg theory $W /\{j\}$. It is therefore of interest to examine further the geometrical representation of this projection in Calabi-Yau manifolds.

We have found above two related ways to connect the $(51,3) Z_{2} \times Z_{2}$ orbifold to the $(27,3)$ one. The first utilized the $Z_{4}^{w}$ projection $(3.10)$, whereas the second utilized the $Z_{2}^{w}$ projection (3.11). The two projections are related by $Z_{2}^{w} \equiv Z_{4}^{j} \otimes Z_{4}^{w}$. Therefore, the substitution of (3.11) for (3.10) corresponds to choosing different elements to generate the same group. However, the difference between the two projections, which is interesting from geometrical view of these potentials, is that in the first case, eq. (3.10), the trivial superfields are left invariant by the action of $Z_{4}^{w}$, whereas with the second choice (3.11), the trivial superfields are twisted by the action of $Z_{2}^{w}$. From a geometric point of view, the addition of the trivial superfields is very natural, as it mimics the structure of $\left(T^{2}\right)^{3}$. In terms of Landau-Ginzburg orbifold there is no need to have the extra trivial superfields, making the $Z_{4}^{w}$ quotient the more natural operation. We note, however, that the fact that twisting of trivial superfields results 
in new models makes the prospects of classifying Landau-Ginzburg orbifolds with $c=9$ more problematic (see however [21]), as we can add any number of such fields to the superpotential. However, the equivalence of such a twist to a twist that closes on the scaling symmetry might facilitate the classification.

\section{Discussion and Conclusions}

The realistic free-fermion models have offered to date the most promising prospect for producing a realistic superstring model. The main reasons to believe that the true string vacuum is located in the vicinity of this class of string vacua is not due to the detailed properties of one of the specific solutions which have been constructed to date, but rather due to the underlying gross structure of these models and their general properties. These include the correlation of three generations with the underlying compactification and the natural standard $S O(10)$ embedding of the weak hypercharge. These models naturally give rise to flavor-universal untwisted moduli, which is not expected to be the case in a generic superstring model. Finally, these models naturally give rise to symmetries that may be required in order to maintain the proton stability. Thus, we see that the realistic free-fermion models naturally contain the necessary ingredients that a realistic string model must contain. The realistic nature of the free-fermion models arises for a very basic reason. They are based on an underlying $Z_{2} \times Z_{2}$ orbifold compactification. It is therefore of vital importance to extend our understanding of this particular class of orbifold compactifications.

The nonperturbative extension of the weakly coupled free-fermion models will surely offer important further insight to their properties. For this purpose we may attempt to use the various $F$ - (and $M-$ ) theory dualities that have been recently discovered. The key point in $F$ theory, compactified to six dimensions, is that the models admit an elliptic fibration in which a Calabi-Yau threefold is identified as a two complex-dimensional base manifold $B$ with an elliptic fiber. A class of elliptically fibered Calabi-Yau three-folds which can be written as $K_{3} \times T_{2}$ modded out with an appropriate $Z_{2}$ symmetry, has been analyzed by Voisin [22] and Borcea [23] (see also [24]). Among these models are those which have an orbifold interpretation. In particular there are $Z_{2} \times Z_{2}$ orbifolds with different $h_{11}$ and $h_{21}$. For example, in $[25]$ the models with $\left(h_{11}, h_{21}\right)=\{(11,11) ;(19,19) ;(51,3)\}$ have been examined in connection with $F$-theory compactifications. These models are constructed as orbifolds of $\left(T^{2}\right)^{3}$ with a generic choice of complex structure for the tori. However, the above $Z_{2} \times Z_{2}$ orbifold models, and many more, can also be obtained from the quartic Landau-Ginzburg superpotential, namely (3.6) with a possible suitable choice of an additional $Z_{4}$ projection. Because of the choice of a square lattice for the tori, rather than having the full $S L(2, Z)$ action on the elliptic fiber, we are restricted to a one-parameter family which leaves $\tau=i$ invariant. This could, however, still be a good $F$-theory vacuum. Alternatively, these models can be studied in five dimensions in terms of compactifications of $M$ theory. In this regard, the key property of the 
$Z_{2} \times Z_{2}$ orbifold that should serve as the basic guide in seeking its possible nonperturbative extension is its basic characteristic property, namely the cyclic permutation symmetry of the twisted and untwisted sectors. It may not be a coincidence that this characteristic property of the $Z_{2} \times Z_{2}$ orbifold, which is clearly of vital phenomenological importance, may also serve as the guiding light in the search for the nonperturbative extension of the phenomenologically realistic free-fermion models.

\section{Acknowledgments}

We are pleased to thank David Morrison and Cumrun Vafa for discussions. This work was supported in part by the Department of Energy under Grants No. DEFG-05-86-ER-40272 and DE-FG03-95-ER-40917. The work of P.B. was supported in part by the National Science Foundation grant NSF PHY94-07194. 


\section{References}

[1] E. Witten, Nucl. Phys. B471 (1996) 135.

[2] I. Antoniadis, J. Ellis, J. Hagelin and D.V. Nanopoulos, Phys. Lett. B231 (1989) 65; see also Phys. Lett. B205 (1988) 459; Phys. Lett. B208 (1988) 209;

J.L. Lopez, D.V. Nanopoulos and K. Yuan, Nucl. Phys. B399 (1993) 654.

[3] A.E. Faraggi, D.V. Nanopoulos and K. Yuan, Nucl. Phys. B335 (1990) 347.

[4] I. Antoniadis. G.K. Leontaris and J. Rizos, Phys. Lett. B245 (1990) 161;

G.K. Leontaris, Phys. Lett. B372 (1996) 212.

[5] A.E. Faraggi, Phys. Lett. B278 (1992) 131; Phys. Lett. B274 (1992) 47; Phys. Lett. B339 (1994) 223.

[6] S. Chaudhuri, G. Hockney and J.D. Lykken, Nucl. Phys. B469 (1996) 357.

[7] H. Kawai, D.C. Lewellen, and S.H.-H. Tye, Nucl. Phys. B288 (1987) 1;

I. Antoniadis, C. Bachas, and C. Kounnas, Nucl. Phys. B289 (1987) 87;

I. Antoniadis and C. Bachas, Nucl. Phys. B298 (1988) 586;

R. Blum, L. Dolan and P. Goddard, Nucl. Phys. B309 (1988) 330.

[8] For reviews and references, see e.g.,

J. Lykken, hep-ph/9511456;

J.L. Lopez, hep-ph/9601208;

K.R. Dienes, hep-th/9602045;

A.E. Faraggi, hep-ph/9707311.

[9] K. Narain, Phys. Lett. B169 (1986) 41.

[10] A.E. Faraggi and J.C. Pati, hep-ph/9712516.

[11] J.C. Pati, Phys. Lett. B388 (1996) 532.

[12] J. Ellis, A.E. Faraggi and D.V. Nanopoulos, hep-th/9709049.

[13] For reviews and references, see e.g., J. Schwarz, hep-th/9607201;

M.J. Duff, hep-th/9608117;

P.K. Townsend, hep-th/9612121;

C. Vafa, hep-th/9702201;

A. Sen, hep-th/9802051.

[14] A.E. Faraggi, Phys. Lett. B326 (1994) 62.

[15] E. Martinec, Phys. Lett. B217 (1989) 431. 
[16] C. Vafa and N. Warner, Phys. Lett. B218 (1989) 51.

[17] A.E. Faraggi and D.V. Nanopoulos, Phys. Rev. D48 (1993) 3288;

A.E. Faraggi, Nucl. Phys. B387 (1992) 239; hep-th/9708112.

[18] First paper in [2];

J.L. Lopez and D.V. Nanopoulos, Nucl. Phys. B332 (1990) 73; Phys. Lett. B251 (1990) 73; Phys. Lett. B256 (1991) 150; Phys. Lett. B268 (1991) 359

A.E. Faraggi, Nucl. Phys. B407 (1993) 57;

A.E. Faraggi and E. Halyo, Nucl. Phys. B416 (1994) 63.

[19] C. Vafa, Mod. Phys. Lett. A4 (1989) 1169;

K. Intriligator and C. Vafa, Nucl. Phys. B339 (1990) 95.

[20] B.R. Greene and M.R. Plesser, Nucl. Phys. B338 (1990) 15.

[21] M. Kreuzer and Harald Skarke, Nucl. Phys. B405 (1993) 305.

[22] C. Voisin, in Journées de Géométrie Algébrique d'Orsay (Orsay, 1992), Astérisque 218 (1993) 273.

[23] C. Borcea, in Essays on Mirror Manifolds, Vol 2,(B. Greene and S.-T. Yau, eds.), International Press, Cambridge, 1997, p. 717.

[24] V. Nikulin, in Proceedings of the International Congress of Mathematicians (Berkeley, 1986), p. 654.

[25] D. Morrison and C. Vafa, Nucl. Phys. B476 (1996) 437;

R. Gopakumar and S. Mukhi, Nucl. Phys. B479 (1996) 260;

J.D. Blum and A. Zaffaroni, Phys. Lett. B387 (1996) 71;

A. Dabholkar and J. Park, Phys. Lett. B394 (1997) 302. 